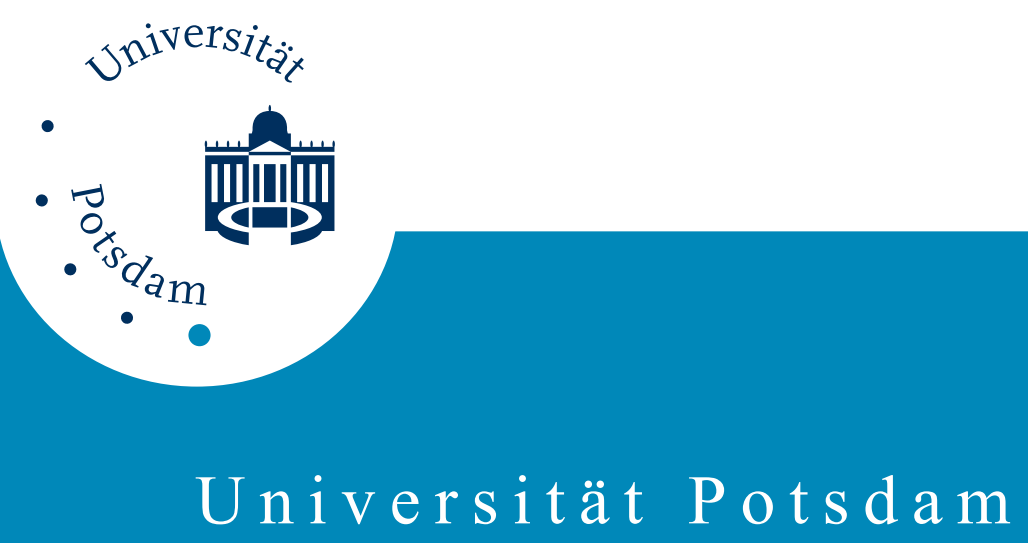

Annette Witt, Alexander Neiman, Jürgen Kurths

Characterizing the dynamics of stochastic bistable systems by measures of complexity

NLD Preprints ; 36 


\title{
Characterizing the dynamics of stochastic bistable systems by measures of complexity
}

\author{
Annette Witt, Alexander Neiman†, Jürgen Kurths ${ }^{\ddagger}$ \\ Arbeitsgruppe Nichtlineare Dynamik an der Universität Potsdam, Pf. 601553, \\ D-14415 Potsdam
}

\begin{abstract}
The dynamics of noisy bistable systems is analyzed by means of Lyapunov exponents and measures of complexity. We consider both the classical Kramers problem with additive white noise and the case when the barrier fluctuates due to additional external colored noise. In case of additive noise we calculate the Lyapunov exponents and all measures of complexity analytically as functions of the noise intensity resp. the mean escape time. For the problem of fluctuating barrier the usual description of the dynamics with the mean escape time is not sufficient. The application of the concept of measures of complexity allows to describe the structures of motion in more detail. Most complexity measures sign the value of correlation time at which the phenomenon of resonant activation occurs with an extremum.
\end{abstract}

\section{Introduction}

Phenomena of noisy bistability are archetypical for wide areas of physics, chemistry and biology. With the classical work of Kramers' [1] theoretical and experimental investigations these phenomena became a field of intensive investigations in nonlinear science (see e.g. [2]). Recently two new nonlinear cooperative phenomena have been discovered for a general class of noisy bistable systems: the phenomenon of stochastic resonance and the phenomenon of resonant activation. The stochastic resonance (SR) occurs in bistable systems which are simultaneously driven by noise and a periodic signal. Tuning the noise intensity, an enhancement of the response of the system to the periodical force becomes possible [3]. This effect appears when the mean escape rate from a well coincides with the frequency of the periodic signal. Recently SR has been studied in terms of information theory $[4,5]$. The phenomenon of resonant activation (RA) takes place in bistable system with fluctuating barriers. In this case the bistable system is driven by additive noise which models the thermal fluctuations and by multiplicative colored noise which leads to fluctuations of the barrier height. Tuning the correlation time of the multiplicative noise, a minimization of the mean escape time from a well can be obtained: for an optimal value of the noise correlation time the mean escape time takes its minimum [6]. Both phenomena underline the nontrivial behavior of nonlinear dynamical systems under the influence of noise.

The study of nonlinear stochastic systems in terms of the Fokker-Planck formalism simply allows to analyze the stationary distributions and the mean-first-passage times for low-dimensional systems. However the detailed dynamical description of motion are difficult to obtain both analytically and numerically. In this paper we therefore apply two other approaches from nonlinear dynamics to study noisy bistable dynamics: distributions of local Lyapunov exponents and methods of symbolic dynamics. The use of these methods is obvious for such systems, because we can naturally introduce a two-symbol alphabet corresponding to the two most probable regions in the phase space of the system.

Such symbol sequences can be characterized by a broad class of so-called measures of complexity. Traditional measures of complexity, such as Shannon entropy or algorithmic complexity, 
are measures of randomness or disorder. Recently, a lot of alternative measures of complexity have been proposed. They yield maximal complexity if the system passes a critical value, e.g. it switches between regular and chaotic behavior. We discuss in case of the classical Kramers problem the relation between these measures of complexity and the traditional measures for describing bistable systems. For the more complex system with the fluctuating barrier we show that the traditional parameters, as mean escape time or global Lyapunov exponents, are not sufficient for a complete characterization of the dynamics and we will explain that measures of complexity give some additional information about the motion of the Brownian particle. This becomes especially important for the analysis of time series obtain from experimental sets when classical quantities become difficult to calculable.

This paper is organized as follows. In the Section 2 we describe the models and discuss the behavior of classical quantities, such as stationary probability density or mean first passage time. The Lyapunov exponents are studied in the Section 3. Section 4 is devoted to measures of complexity. Finally, the results are discussed in Section 5.

\section{The models}

We treat the very popular model of an over-damped Kramers oscillator

$$
\dot{x}=-\frac{d U(x)}{d x}+\sqrt{2 D} \xi(t)
$$

with the symmetric potential

$$
U(x)=-\frac{1}{2} x^{2}+\frac{1}{4} x^{4},
$$

where $\xi(t)$ is zero-mean Gaussian white noise,

$$
<\xi(t) \xi\left(t^{\prime}\right)>=\delta\left(t-t^{\prime}\right) .
$$

The stochastic differential equation (SDE) (1) describes the noisy dynamics of an over-damped Brownian particle in the double-well potential $U$. The stationary probability density is yielded as the solution of the appropriate Fokker-Planck equation (FPE)

$$
\partial_{t} p(x, t)=D \partial_{x x} p(x, t)+\partial_{x}\left[U^{\prime}(x) p(x, t)\right]
$$

which has the steady-state solution

$$
p_{s}(x)=N \exp \left(-\frac{U(x)}{D}\right)
$$

where $N$ is the normalization constant $N^{-1}=\int_{-\infty}^{+\infty} \exp \left(-\frac{U(x)}{D}\right) d x$. The stationary distribution has two maxima corresponding to the stable states of the appropriate dynamical system and a minimum at the origin referring to the saddle point $x=0$.

The quantity of crucial interest in noisy bistable dynamics is the mean first passage time (MFPT) of the top of the potential. This quantity can be obtained from the Pontryagin equation [7]:

$$
D \frac{d^{2} T(x)}{d x^{2}}-U^{\prime}(x) \frac{d T(x)}{d x}=-1 .
$$

As boundary conditions for the $T(x)$ we have chosen an absorbing boundary condition at the top of the barrier $(x=0)$ and a reflecting boundary condition at $x \rightarrow-\infty$ :

$$
T(x=0)=0, \quad \frac{d T}{d x}(x \rightarrow-\infty)=0 .
$$


The mean escape time from the left potential well is then the solution of Eq.(6) with the specified initial conditions (7) [8]:

$$
T_{0}(D)=\frac{1}{D} \int_{-1}^{0} d y \exp [U(y) / D] \int_{-\infty}^{y} \exp [-U(z) / D] d z .
$$

This quantity diverges when the noise intensity tends to zero. For small noise intensities $D<\Delta U$, where $\Delta U$ is the barrier height, $T_{0}(D)$ follows the well-known Arrhenius law:

$$
T_{0}(D) \propto \exp (\Delta U / D),
$$

whereas for strong noise $(D>\Delta U)$ the Arrhenius law is not longer valid. This transition does not disturb the stationarity of the probability density and becomes visible if we calculate the second derivative of the quantity $T_{0}(D)$. It vanishes at $D \approx 0.6[9]$.

The second objective studied here are bistable systems simultaneously driven by Gaussian white noise and multiplicative colored noise. This class of models refers to a topic of high recent interest in the analysis of stochastic nonlinear systems, namely to the problem of surmounting fluctuating barriers [6]. It describes a variety of physical situations e.g. biological transport [10], or the dynamics of dye laser [11]. The non-trivial phenomenon which occurs in such systems is the so-called resonant activation: there is an optimal value of correlation time of multiplicative noise at which the mean escape time from a well takes its minimal value. The model we study here is the same as the previous one but with an additional colored noise source which modulates the barrier height:

$$
\dot{x}=-\frac{d U(x)}{d x}+\sqrt{2 D} \xi(t)+x y(t),
$$

$y(t)$ is exponentially correlated Gaussian noise which is modeled by an Ornstein-Uhlenbeck process. Thus the two-dimensional Markov process is described by the following set of SDEs

$$
\begin{aligned}
\dot{x} & =x-x^{3}+\sqrt{2 D} \xi(t)+x y, \\
\dot{y} & =-\frac{y}{\tau}+\frac{\sqrt{2 Q}}{\tau} \theta(t),
\end{aligned}
$$

where $\xi(t)$ and $\theta(t)$ being statistically independent Gaussian white noise processes:

$$
<\xi(t) \xi(0)>=<\theta(t) \theta(0)>=\delta(t), \quad<\xi(t) \theta(0)>=0 .
$$

The analytical study of this model has been recently done by Hänggi and co-workers [12], whose analogous simulations of Eqs. (11) show the phenomenon of resonant activation [13]. They have explained that the probability density of the Brownian particle is increasingly concentrated in the tales of the potentials with growing inverse correlation time $1 / \tau$.

In Fig. 1 we show the numerical results of the mean escape time as a function of the inverse correlation time $1 / \tau$. The magnitude of colored noise is constant while $\tau$ varies:

$$
\sigma^{2}=Q / \tau=\text { const }
$$

As can be seen from the figure, the phenomenon of resonant activation takes place at the correlation time $\tau \approx 1$ at which the mean escape time takes its minimum. These results are in agreement with the analog simulations of [13].

\section{Lyapunov exponents in noisy bistable systems}

In nonlinear dynamics Lyapunov exponents play a very important role as indicators of chaos in the system under study. Although Lyapunov exponents are well defined for stochastic systems 


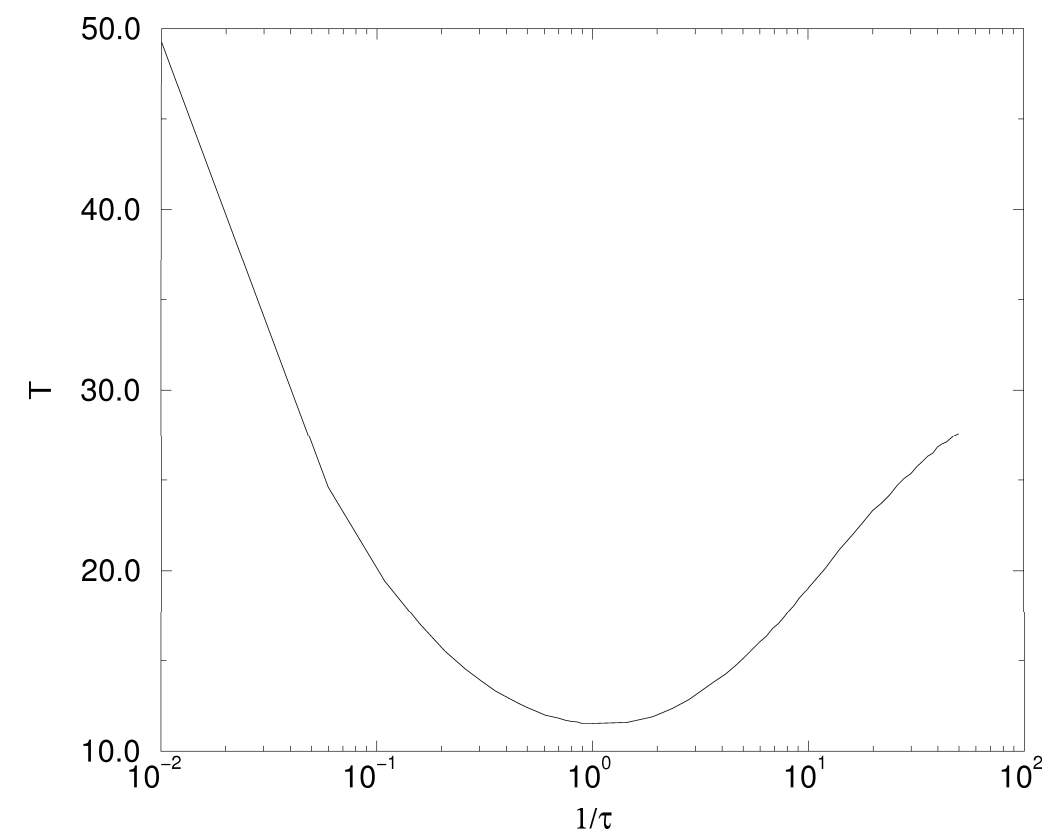

Figure 1: The mean escape time as a function of $\tau$ for the model (10). Other parameters are: $\sigma=1.0, D=0.1$

(cf. [14]), they do not longer refer to the Kolmogorov-Sinai entropy [15]. However, as we are showing below, the Lyapunov exponent and especially the local Lyapunov exponents might reflect main features of the over-damped stochastic dynamics.

Firstly we deal with the Kramers oscillator. Let us consider the separation $\delta x(t)$ between two trajectories of a Brownian particle, whose initial conditions differ by $\delta x_{0}$ but with the same noise $\xi(t)$. The SDE for the separation reads:

$$
\dot{\delta x}(t)=-\frac{d^{2} U(x)}{d x^{2}} \delta x(t)=\lambda(x, t) \delta x(t),
$$

where the quantity $\lambda(x, t)$ is called local (or instantaneous) Lyapunov exponent ${ }^{1}$. The Lyapunov exponent is then defined as the long-time average of the local exponent [15]:

$$
\Lambda=\lim _{t \rightarrow \infty} \frac{1}{t} \int_{0}^{t} \lambda(x, s) d s=\lim _{t \rightarrow \infty} \frac{1}{t} \log \frac{|\delta x(t)|}{\left|\delta x_{0}\right|} .
$$

The long-time average in (15) can be replaced by an averaging with respect to the stationary probability density (5):

$$
\Lambda=<\lambda(x)>=\int_{-\infty}^{+\infty} \lambda(x) p_{s}(x) d x .
$$

For the specified potential (2) we immediately obtain

$$
\Lambda=1-3<x^{2}>\text {. }
$$

\footnotetext{
${ }^{1}$ The simple notion of the local Lyapunov exponent is valid for our one-dimensional case only. If the dimension of the phase space is greater than 1, the direction of perturbations has to be taken into account, too
} 
The second moment $\left\langle x^{2}\right\rangle=\int_{-\infty}^{+\infty} x^{2} p_{s}(x) d x$ might be expressed via the parabolic cylinder functions (see [16]). Finally we obtain for the Lyapunov exponent

$$
\Lambda(D)=1-3 \sqrt{\frac{D}{2}} \frac{D_{-3 / 2}(-1 / \sqrt{2 D})}{D_{-1 / 2}(-1 / \sqrt{2 D})},
$$

where $D_{c}(z)$ is the parabolic cylinder function [17].

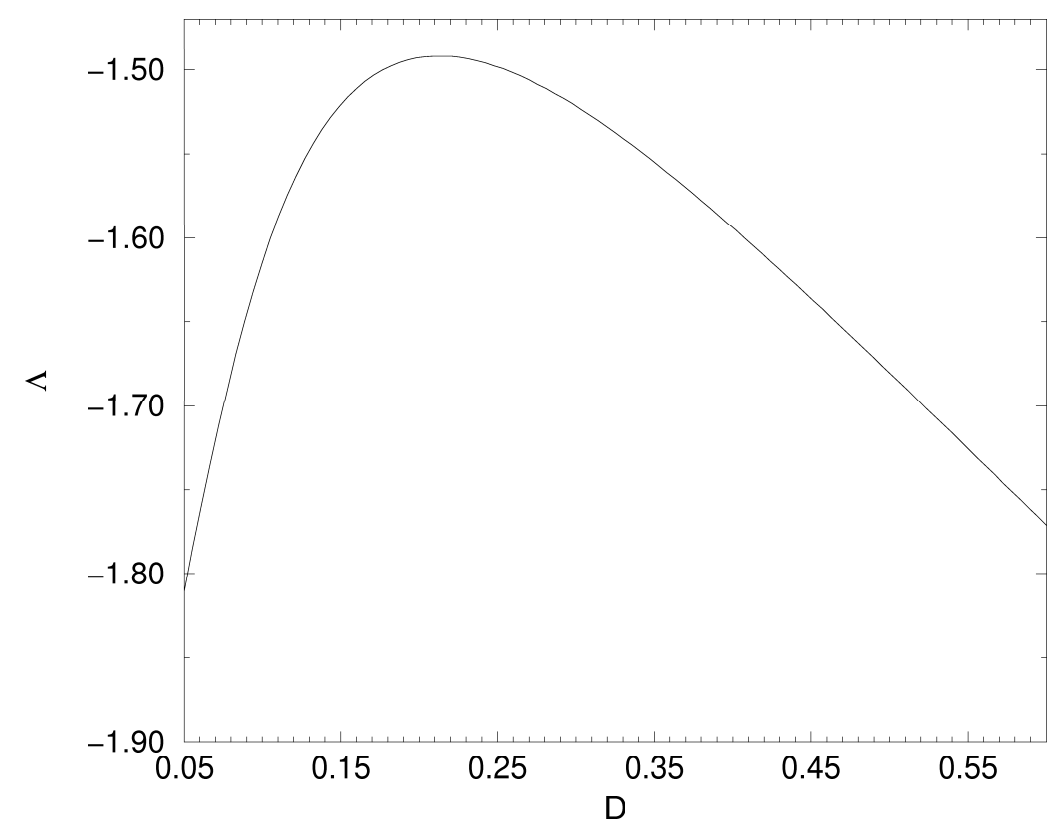

Figure 2: The Lyapunov exponent vs. the noise intensity for the model (1)

¿From calculating the dependence of $\Lambda$ on the noise intensity $D$ (Fig. 2), we find that two distinct regimes may be observed and the Lyapunov exponent takes its maximum value for a certain noise intensity $D^{(m)} \approx 0.22 \ldots$. Now, we discuss the reason for this extremum. This maximum is a sign of noisy bifurcation taking place in this system [9]. The Lyapunov exponent is always negative due to noise-induced global stability [18]. Therefore, at the bifurcation points of noisy dynamical systems the Lyapunov exponent does not vanish but has a smooth extremum [9].

To discuss this aspect in more detail, let us consider the probability density $q(\lambda)$ of the local Lyapunov exponents. This function can be obtained directly from the expression for the stationary probability density $p_{s}(x)(5)$ by using the nonlinear transformation $\lambda=1-3 x^{2}$ :

$$
q(\lambda)=\frac{N}{\sqrt{3}}(1-\lambda)^{-1 / 2} \exp \left\{\frac{1}{12 D}\left[2(1-\lambda)-(1-\lambda)^{2} / 3\right]\right\} .
$$

The local extrema $\lambda_{1,2}$ of this distribution are determined from its first derivative which yields:

$$
\lambda_{1,2}=-\frac{1}{2} \mp \frac{3}{2} \sqrt{1-4 D} .
$$


It is easy to see that for $D<\frac{1}{4}$ the probability density for the local Lyapunov exponent has two local extrema: the maximum at $\lambda_{1}=-\frac{1}{2}-\frac{3}{2} \sqrt{1-4 D}$ and the minimum at $\lambda_{2}=-\frac{1}{2}+\frac{3}{2} \sqrt{1-4 D}$. For $D>\frac{1}{4}$ there is no local extrema, hence the noise intensity $D^{(c)}=\frac{1}{4}$ is the critical one. The stationary probability density for the local Lyapunov exponents is shown in Fig. 3 for three cases of noise intensity: $D<D^{(c)}, D=D^{(c)}$ and $D>D^{(c)}$. For small noise intensities the distribution of the local Lyapunov exponent has the maximum concentrating around negative value $\lambda_{1}$. The

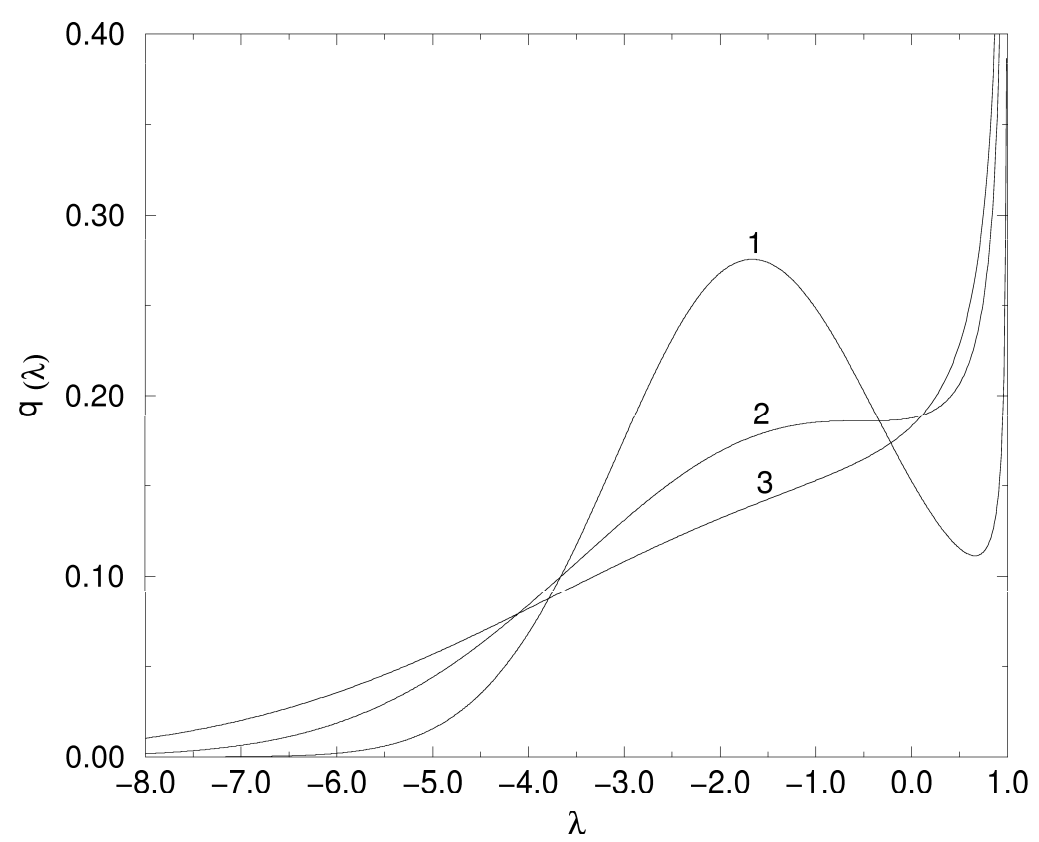

Figure 3: The probability density of the local Lyapunov exponent (cf. Eq. 19) for different values of noise intensity: $D<D^{(c)}(1) ; D=D^{(c)}(2) ; D>D^{(c)}(3)$.

absolute value of $\lambda_{1}$ corresponds to the characteristic rate for probability to equilibrate within a well, while the region of positive $\lambda$ refers to hoppings between potential wells. For large noise there is a long tail in the negative branch of $\lambda$ and no extrema occur.

Note that the critical value of noise intensity $D^{(c)}$ at which this bifurcation-like transition occurs is equal to the barrier height $\Delta U$ of the potential. This value is shifted a little bit relatively to that at which the Lyapunov exponent takes its maximum $D^{(m)}$. This is, however, a usual situation in noisy nonlinear systems where we cannot speak about a bifurcation point, but rather about a bifurcation region because different dynamical and probabilistic quantities display shifts of transition points between the two regimes [9].

Thus we have shown that in stochastic bistable system (1) the Lyapunov exponent takes its maximum for a certain noise intensity. This maximum refers to a qualitative change in the shape of the probability distribution of local Lyapunov exponents. This change occurs if the value of noise intensity equals to the barrier height. Therefore the above mentioned transition corresponds to the destruction of stochastic bistability. For the weak noise $(D<\Delta U)$ we have well separated two characteristic time scales. The first, long-range time scale is the Kramers time and corresponds to the random hoppings between potential wells. The second one refers to the short-range fluctuations within each well. The distribution of the local Lyapunov 
exponent reflects this situation having local maximum which just corresponds to the short-range characteristic time scale. For the large enough noise strength $(D>\Delta U)$ the average time scale of switching becomes comparable with those of motion within the wells. Hence, the Kramers theory is no longer valid. As a result the distribution of the local Lyapunov exponents has no extrema.

For the second model with the fluctuating barrier (10) an exact analytical treatment is only possible in the limiting cases of very slow and very fast colored noise [12]. Here we study this model by means of numerical simulations of the appropriate SDE (10). The dependence of the Lyapunov exponent on the noise intensity is qualitatively the same as in the previous model. If the Lyapunov exponent is regarded as a function of the correlation time of the barrier fluctuations $\tau$, it exhibits a crossover behavior (see Fig. 4). For very fast barrier fluctuations the intensity of colored noise $Q$ vanishes and the Lyapunov exponent saturates to that value obtained in the previous case without barrier fluctuations. In the case of very slow barrier fluctuations, when correlation time is longer than the mean escape time from a potential well, colored noise $y(t)$ forces potential to change adiabatically. For large negative deviations of $y(t)$, the system can be mono-stable for a long time which leads to a decrease of the Lyapunov exponent. The crossover behavior refers to the values of correlation times at which the phenomenon of resonant activation occurs. The probability density of the local Lyapunov exponent $\lambda(t)=1+y(t)-3 x^{2}(t)$

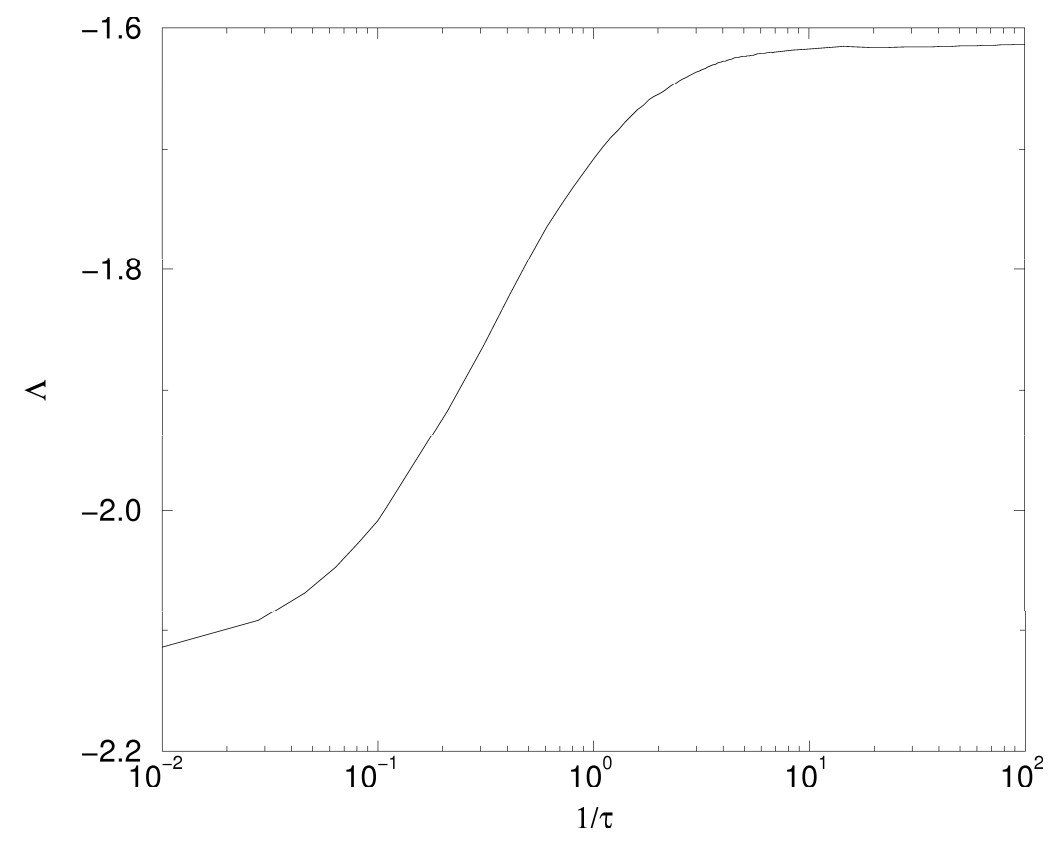

Figure 4: The Lyapunov exponent versus $\tau$ for the model (10). Other parameters are: $D=0.1$, $\sigma=1$.

again provides more information as demonstrated in Fig. 5 For very slow barrier fluctuations the probability density of the local Lyapunov exponent (curve 3) has a small bump and a maximum. The maximum refers, as in the case with additive white noise only, to the rate for probability to equilibrate within a well, while the bump corresponds to mono-stable dynamics when the colored noise $y(t)$ has large negative deviations. Note that in contrast to the first model, where the range 
of possible values of the local Lyapunov exponents was bounded by the positive value +1 , for the second model this boundary is washed out by barrier fluctuation $y(t)$. With decreasing correlation time of the barrier fluctuations the positive tail of the distribution of the local Lyapunov exponents grows leading to an increase of the averaged Lyapunov exponent.

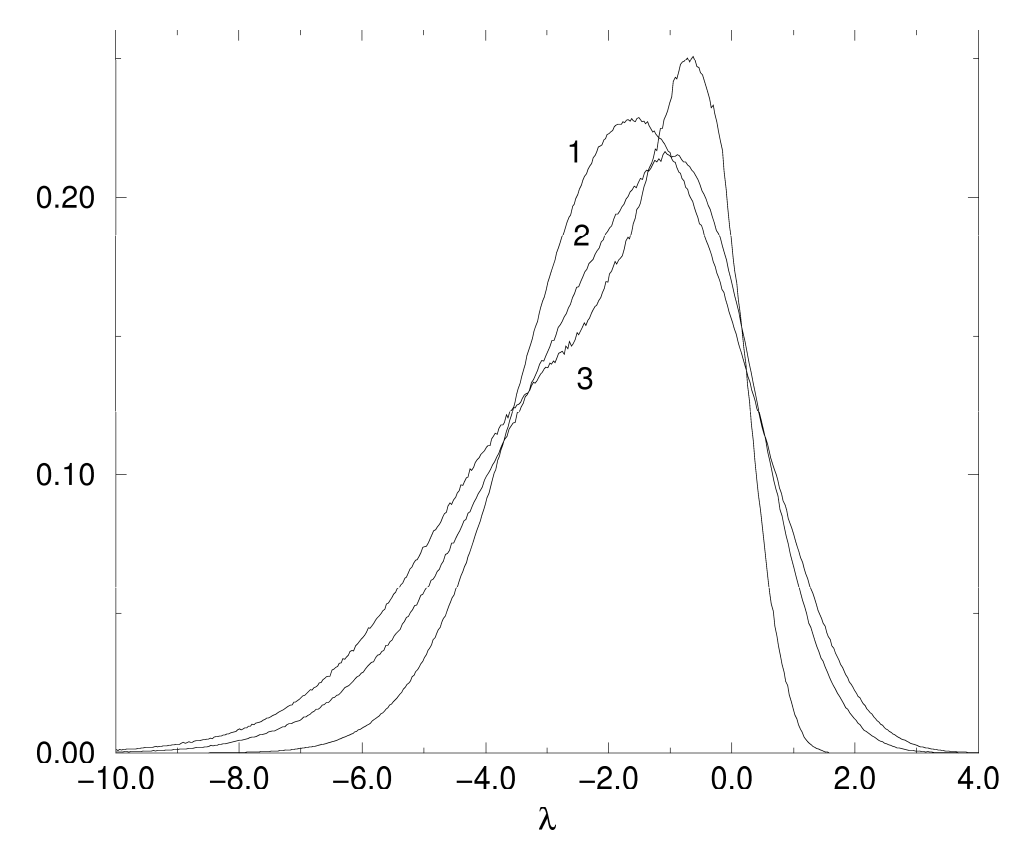

Figure 5: The probability density of the local Lyapunov exponent for the system (10) for different values of correlation time: $\tau=100.0(1) ; \tau=1.0(2) ; \tau=0.01$ (3) with $D=1.0$ and $\sigma=1$.

\section{Measures of complexity}

In this section we represent some measures of complexity [19] and show whether they are useful to describe the dynamics of bistable systems in some detail[22].

All these measures of complexity are defined for symbol sequences. Therefore, we have firstly to coarse-grain the trajectories of the considered systems in phase-space and time.

The time axis is equi-distantly scanned with a time step $\Delta$. The spatial position is reduced to the information in which half of the double-well potential the particle is situated. This way we transform each trajectory $x(t)$ into a symbol sequence

$$
s_{\Delta}(k)=\operatorname{sign}(x(k \Delta))
$$

which makes an application of the concept of measures of complexity possible.

In the following we introduce some of these measures. Their properties are explained for a few elementary types of symbol sequences:

1. periodic symbol sequences with period $p$.

2. purely random sequences: The occurrence of the elements is completely uncorrelated. Here, only such sequences with uni-probable symbols are regarded, as in case of a coin flip. 
3. Markov processes of order $k$ : The structure of the symbol sequence is completely described with the distribution of substrings of length $k+1$.

\subsection{Special measures of complexity}

\section{Shannon entropy}

The traditional quantity for characterizing a symbol sequence is the Shannon entropy [23].

The Shannon entropy of $n$-th order $H_{n}$ is based on the probability distribution of length- $n$ substrings $s^{n}$ (words of length $n$ ) of the symbol sequence:

$$
H_{n}=-\sum_{s^{n} \in A^{n}, p\left(s^{n}\right)>0} p\left(s^{n}\right) \log _{2} p\left(s^{n}\right),
$$

where $A^{n}$ denotes the set of all length- $n$ words.

$H_{n}$ measures the average number of bits needed to specify an arbitrary word of length $n$ in a sequence $S$. Their differences

$$
\begin{aligned}
h_{n} & =H_{n+1}-H_{n} \\
h_{0} & =H_{1}
\end{aligned}
$$

quantify the information needed to determine the $(n+1)^{t h}$ symbol of an arbitrary word of a given sequence if the first $n$ symbols are known. The Shannon entropy of the system is then defined as the limit of the $h_{n}$ :

$$
h=\lim _{n \longrightarrow \infty} h_{n}
$$

It describes the mean information contents per symbol. For the $h_{n}$ it holds:

1. For period- $p$ - sequences, all $h_{n}$ with $n \geq p$ vanish.

2. Due to Eqs. (22) and (23) all $h_{n}$ are equal to $n$ in case of purely random symbol sequences.

3. For $k^{t h}$ order Markov processes the entropy differences reach the value of the entropy with $n=k: h_{n}=h_{k}$ for $n>k$.

These properties are similar to those of algorithmic complexity; both measures reach their maximum in case of completely uncorrelated symbol chains. Therefore they are called measures of randomness. For describing dynamical phase transitions, other approaches are necessary.

Effective Measure Complexity

It is of special meaning, how the Shannon entropy differences converge to the Shannon entropy. This behavior can be analyzed in more detail in the approach of dynamical entropies [15] where scaling laws of the $H_{n}$-s are described. Another approach is the Effective Measure Complexity (EMC) introduced by Grassberger [24] as a special parameter which characterizes this convergence behavior:

$$
E M C=\sum_{n=1}^{\infty} n\left(h_{n-1}-h_{n}\right)
$$

Small (large) $E M C$ corresponds to fast (slow) convergence. For the exemplary models EMC behaves as:

- If periodic behavior of period $p$ is considered, this implies

$$
E M C=\log p
$$

In special case of a constant symbol sequence $(p=1)$, EMC vanishes.

- For purely random sequences one finds $h_{i}=1$ for all $i$ which leads to a vanishing $E M C$. 
- For $k$-th ordered Markov processes the EMC is reached for the first $k$ summands of Eq. 26.

For complex processes (e.g. with long-range memory) infinitely many summands of the $E M C$ can be positive, moreover it might be that their sum diverges. Diverging complexity assigns to dynamical phase transitions e.g. transitions from order to chaos via period doubling [19].

Fluctuation complexity $\sigma_{\Gamma}^{2}$

Another way to characterize the complexity of dynamical systems is based on the evaluation of local information gain and information loss [25]. Bates and Shepard [19] have introduced a complexity measure called fluctuation in net information gain which is defined as

$$
\begin{aligned}
\sigma_{\Gamma}^{2} & =<\Gamma^{2}>-<\Gamma>^{2} \\
& =\sum_{i, j} p_{i j}\left(\log \frac{p_{i}}{\left(p_{j}\right.}\right)^{2}
\end{aligned}
$$

where $i$ and $j$ are generalized states of a dynamical system. In case of symbol sequences when words of length $n$ are considered, this quantity reads

$$
\sigma_{\Gamma}^{2}(n)=\sum_{\substack{w \in \mathcal{A}^{n-1} \\ s_{1}, s_{2} \in \mathcal{A}}} p\left(s_{1} \cdot w \cdot s_{2}\right)\left(\log \frac{p\left(w \cdot s_{2}\right)}{p\left(s_{1} \cdot w\right)}\right)^{2} .
$$

Thereby $s_{1} \cdot w$ notates the concatenation of the symbol $s_{1}$ and the word $w$.

Some of the essential properties of the fluctuation in net information gain are

- In case of periodic behavior $\sigma_{\Gamma}(n)$ vanishes independently of the prime period $p$ if sufficient long words are considered $(n \geq p)$.

- For uncorrelated sequences the fluctuation complexity does not depend on the word length $n$. In case of purely random behavior the complexity vanishes: $\sigma_{\Gamma}^{2}=0$.

- The fluctuation complexity $\sigma_{\Gamma}(n)$ behaves in dependence on $n$ for $k$-th order Markov processes periodically with period $k$.

The fluctuation complexity indicate special phase transitions in dynamical systems as for the symbol sequences of band merging points.

\section{Complexity of $\epsilon$-Machines}

It is well known that each finite-order Markov process can be equivalently represented by a stochastic automaton [26]. Crutchfield and Young [27] suggested a technique which constructs for a given symbol sequence a stochastic automaton which can $\epsilon$-equivalently reproduce the given sequence. The $\epsilon$-complexity $C_{\epsilon}$ of the (original) sequence is then quantified by the Shannon entropy with respect to the probabilities of these automaton states. The $\epsilon$-complexity is based on state probabilities on the level of the automaton, which refer to both transition probabilities and state probabilities on the level of the symbol sequence.

- The automaton constructed for the periodic symbol sequence (..00100100 $100100 \ldots)$ (Fig. 6) contains of a closed loop composed of 3 final automaton states which are occupied with same probability. The $\epsilon$-complexity is then $C_{\epsilon}=\log 3$. Generally, all period- $p$ sequences lead to $\epsilon$-complexity of $\log p$.

- In case of purely random sequences the automaton consist only of one state which implies vanishing complexity.

- For a $k$-th ordered Markov process an automaton is constructed which reproduces the exact structure of the original sequence. The complexity is evaluated between 0 and $k+1$. 


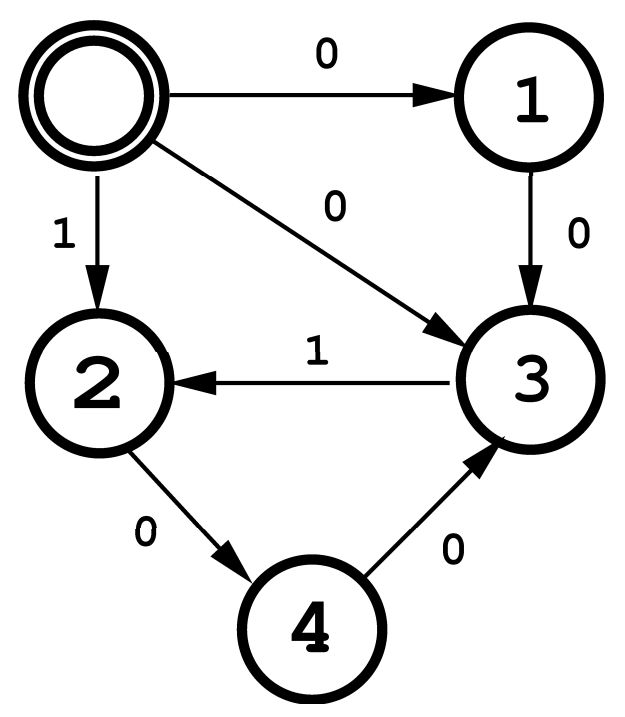

Figure 6: $\epsilon$-machine for a period-3-sequence

For more general structured symbol sequences the $\epsilon$-machine can only approximatively reproduce the given sequence.

The $\epsilon$-complexity indicates phase transitions as the accumulation point of the period doubling cascade or band merging [19].

In case of symbol sequences induced by nonlinear deterministic dynamics, the properties of the non-traditional measures of complexity as $E M C, \sigma_{\Gamma}(n)$ and $C_{\epsilon}$ are detailed studied [19]. These measures indicate qualitative changes in the dynamics in different manner; so $E M C$ and $C_{\epsilon}$ mark the accumulation points of period doubling, whereas the fluctuation complexity points out band merging. Further, this measures are successfully applied for different problems in data analysis $[20,21]$. Therefore, it is promising to use these measures for characterizing complex stochastic dynamics.

\subsection{Results}

\subsubsection{The over-damped Kramers' oscillator}

The corresponding symbol sequences have a rather simple structure: Due to the uncorrelated noise source and the fact that the separation point of the two areas defining the symbols is a saddle point, the symbol sequences have only a one time step memory, i.e. they all can be understood as Markov processes of first order. These Markov processes are completely described by the transition probabilities $p(0 \rightarrow 0)=p(1 \rightarrow 1)=0.5(1+Z)$ and $p(1 \rightarrow 0)=p(0 \rightarrow 1)=0.5(1-Z)$ as well as the additional condition $p_{0}=p_{1}=0.5$. $Z$ is related to the MFPT under the special coarse graining of the time axis $Z=\exp \left(-2 \Delta / T_{0}(D)\right)$.

The knowledge of all transition probabilities enables an analytic calculation of all complexity measures.

For the Shannon Entropy we yield:

$$
h=h_{2}=0.5\left\{(1+Z) \log _{2}(1+Z)+(1-Z) \log _{2}(1-Z)\right\},
$$

i.e. in case of a fixed time step $\Delta$ an increase of the noise intensity leads to higher entropy, the 


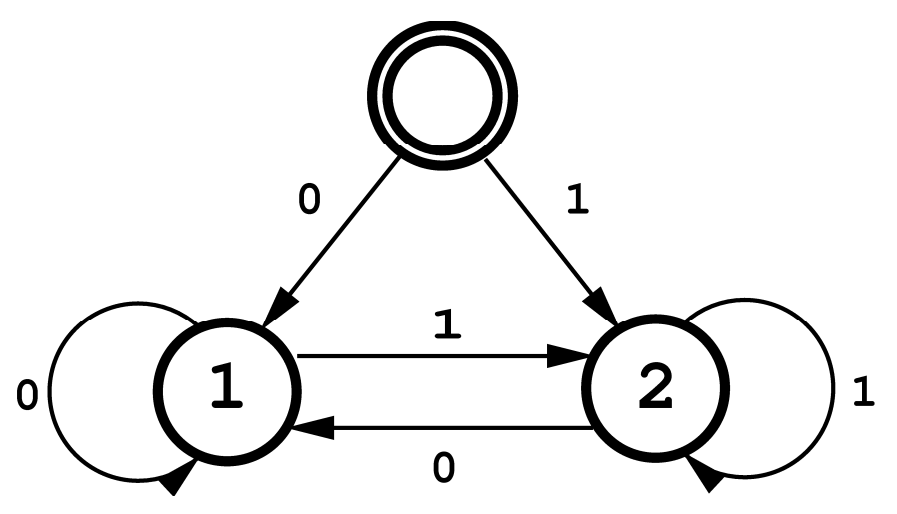

Figure 7: $\epsilon$-machine for the sequences of the over-damped Kramers oscill ator

fluctuations of the particle between the two tales become more disordered. Furthermore, if the noise intensity is fixed and the time step $\Delta$ is raised, the entropy is growing either.

The Effective Measure Complexity simplifies in this special case to

$$
E M C=H_{1}-h_{1}=1-h_{1}=\left\{0.5(1+Z) \log _{2}(0.5(1+Z))+(1-Z) \log _{2}(0.5(1-Z))\right\}
$$

If this complexity is regarded as a function of noise intensity it is monotonically decreasing. This behavior reflects the increase of the disorder in the motion of the particle.

The fluctuation in net information gain shows a completely different behavior. It depends on $Z$ as

$$
\sigma_{\Gamma}^{2}=0.5(1+Z)(1-Z)\left(\log _{2} \frac{(1-Z)}{(1+Z)}\right)^{2}
$$

and leads similar to the Lyapunov exponent to a one-humped behavior. The maximum at $Z_{\max } \approx 0.834$ depends on the noise intensity as well on the time step $\Delta$. Small noise intensities induce symbol sequences with long constant sub-series which leads to small values of $\sigma_{\Gamma}^{2}$ whereas symbol sequences for large noise intensities are almost completely uncorrelated and are, therefore, characterized by small values of $\sigma_{\Gamma}^{2}$ as well.

Due to the special first-order-Markov structure of the symbol sequences the corresponding $\epsilon$ machines are built uniformly (cf. Fig. 7), only the probabilities of the edges differ (in dependence on $Z$ ). The symmetric structure of the automata reflects the symmetry of the potential. The $\epsilon$-complexity is a constant over $D$ and $\Delta, C_{\epsilon}=1$. In the limit for $D \rightarrow \infty$ the structure of the sequence is purely random which leads to an automaton with only one state and a vanishing complexity.

\subsubsection{The system with fluctuating barrier}

The symbol sequences of this system (10) are more complicated structured as the above ones. They inherit long-time correlations of the term which is controlling the fluctuations of the barrier. So, we can find in dependence on the correlation time higher Markov orders. In case of these more complicated dynamics, the transition probabilities cannot be calculated analytically. Therefore we have to analyze results of numerical simulations. The different Shannon entropy differences $h_{n}$ (Fig. 8) clearly show that the convergence to the Shannon entropy $h$ is accelerated with growing $1 / \tau$, especially for $1 / \tau>1$ the limit $h$ is reached with $h_{1}$, i.e. the symbol sequences can also be understood as a Markov process of first order as in case of the system with fixed barrier. For smaller values $1 / \tau$ the symbol sequences have to be interpreted as higher order Markov processes.

If only the limit of the Shannon entropy differences is considered, one finds maximum entropy in case of correlation time $\tau=1$. At this parameter value the symbol sequences contain maximum information per symbol. 


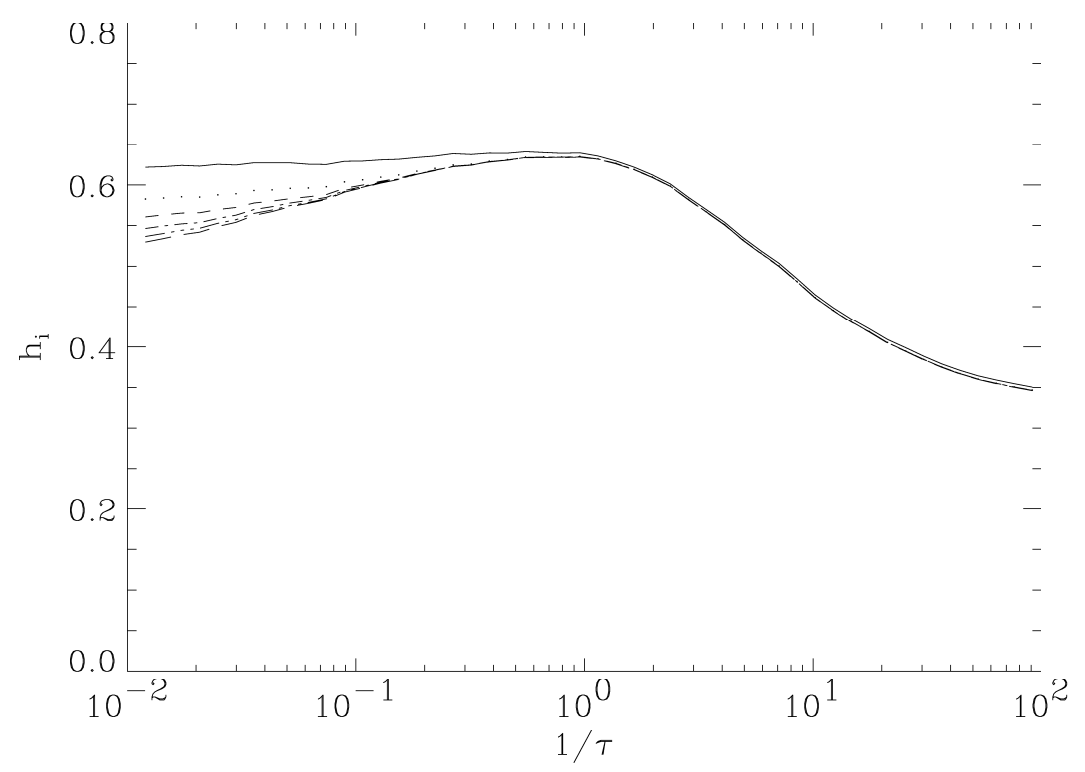

Figure 8: Shannon entropy differences of different order in dependence on the inverse correlation time $1 / \tau$ for the model (10): full line $-h_{1}$, dotted $h_{2}$, dashed $h_{3} \ldots$

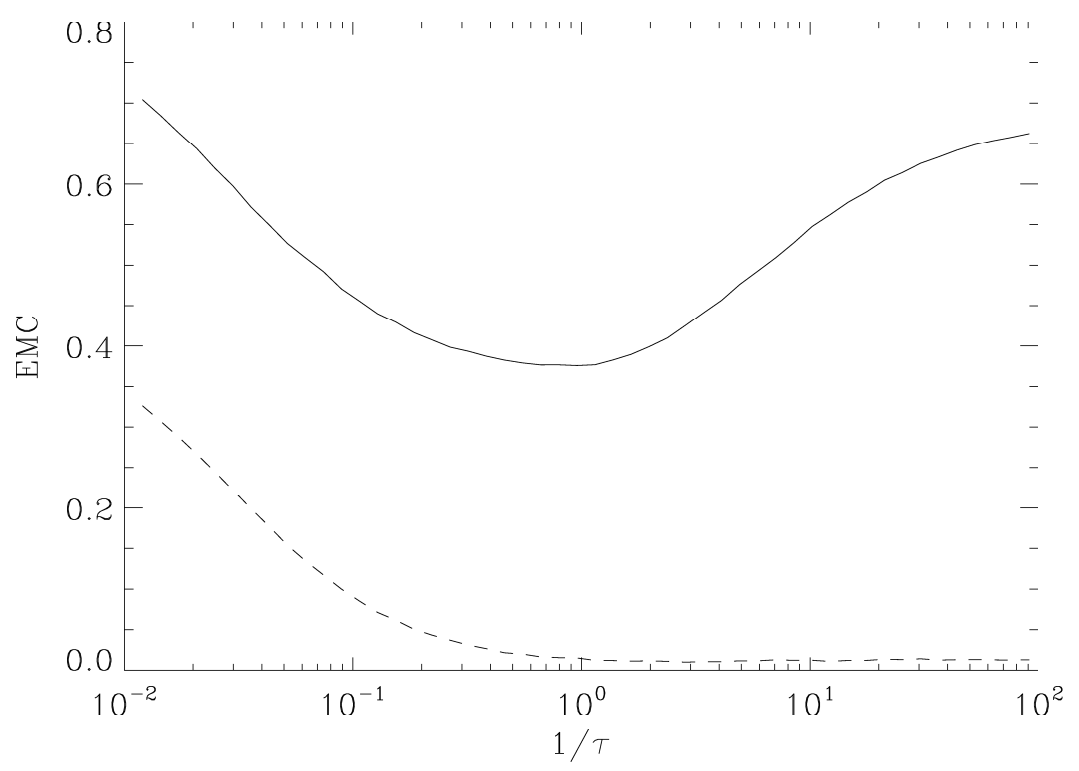

Figure 9: $E M C$ and $\widehat{E M C}$ (dashed line) in dependence on the inverse c orrelation time $1 / \tau$ for the model (10) 


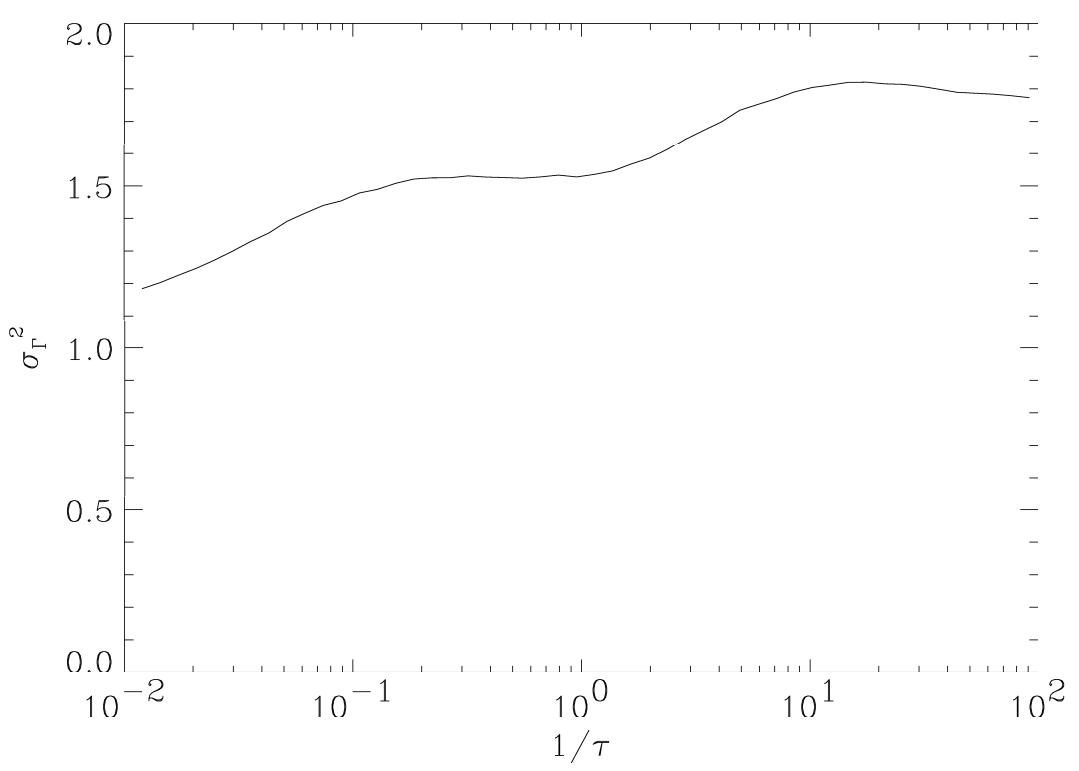

Figure 10: SBC in dependence on the inverse correlation time $1 / \tau$ for the model (10)

To study the influence of the first $h_{i}$ on the $E M C$, we also calculate a modified $E M C$, $\widehat{E M C}=E M C-\left(h_{0}-h_{1}\right)=\sum_{n=2}^{\infty} n\left(h_{n-1}-h_{n}\right)$, where the first summand $\left(h_{0}-h_{1}\right)$ is neglected. The behavior of these measures reflect that the convergence speed of the Shannon entropy differences $h_{n}, \quad n \geq 1$ is accelerated with growing $1 / \tau$ (Fig. 9). For $1 / \tau>1$ the Shannon entropy of the system is well approximated by $h_{1}$ which leads to nearly vanishing $\widehat{E M C}$. The original $E M C$ indicates lowest complexity for $\tau=1$, because the $h_{n}$ (including $n=0$ ) are all near the limit $h$. Due to finite size effects, the direct estimation of Markov order from the $h_{i}$ is difficult to obtain reliably.

A quite different behavior is exhibited by the variation in net information gain (Fig. 10). Qualitative changes in the shape are located at $1 / \tau=0.2$ (transition from increasing to plateaulike behavior), $\tau=1.0$ (transition to increasing behavior) and $1 / \tau=15$ (local maximum). These extrema mark alterations in the system properties: As explained above, there are two typical characteristics of the symbol sequences which change for small inverse correlation times $(0<1 / \tau<1)$ : on the one hand the Shannon entropy is growing and on the other hand the Markov-order is decreasing. The increase of the entropy, which reflects a transition from a nearly constant to a disordered symbol sequence, effects a growth of the complexity, whereas the decrease of the Markov order causes the opposite. For $1 / \tau<0.2$ the growing of the entropy is dominating, whereas for $0.2<1 / \tau<1$ the balance of both influences leads to a nearly constant complexity. For inverse correlation times $1 / \tau>1$, the symbol sequences can be regarded as first order Markov processes and therefore the behavior of the complexity is the same as for the first model with decreasing noise intensity and depends only on the entropy. Therefore, the maximum around $1 / \tau=15$ corresponds to the maximum of the fluctuation complexity for the classical Kramers system.

The $\epsilon$-complexity shows a decreasing behavior (Fig. 10). This reflects the above mentioned fact that the sequences can be approximated by finite-order Markov-processes, whose order is raising with growing $1 / \tau$. Same values of $\epsilon$-complexity for different correlation times mark that the corresponding machines have the same topology; different probabilities of the knots are not 


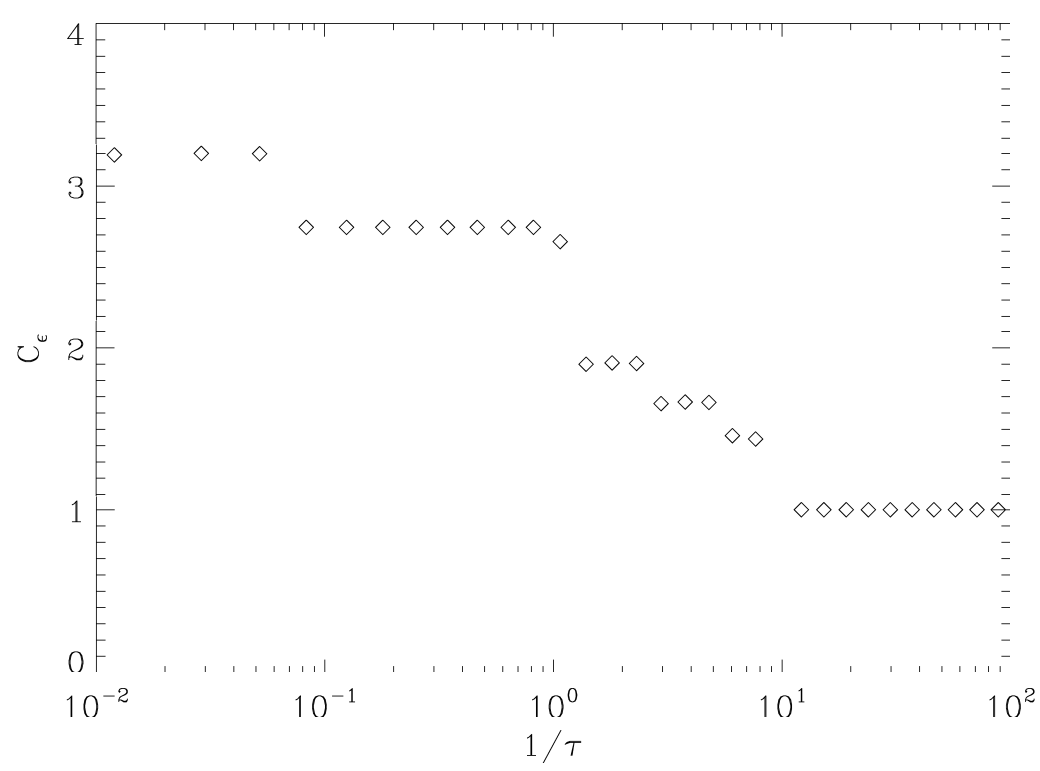

Figure 11: Complexity of $\epsilon$-machines in dependence on the inverse correlation time $1 / \tau$ for the model (10)

considered by $\epsilon$-complexity. So, the six visible plateaus refer to decreasing Markov orders. It should be mentioned that the approximating machines for large values of $1 / \tau$ are the same as in the Kramers case, since the fluctuations of the barrier are almost uncorrelated.

\section{Conclusion}

We have applied the concepts of local Lyapunov exponents and symbolic dynamics for understanding two types of stochastic bistable systems, the simple over-damped Kramers oscillator and an extended model with fluctuating barrier. Using these techniques, we could explain the dynamics of the considered processes in more detail. Our special findings are:

1. For the simple model, the over-damped Kramers oscillator, we can calculate all quantities of interest analytically: we have found a one-humped behavior of the Lyapunov exponent in dependence on the noise intensity. The local Lyapunov exponents provide a more detailed understanding of the dynamics: their distribution reflects the breakdown of the stochastic bistability by growing noise intensity. The noise intensity $D^{(C)}$, at which the maximum of the Lyapunov exponent $\Lambda(D)$ is reached refers to this transition.

Due to the symmetric first order Markov structure of the coarse-grained trajectories, all measures of complexity can be rewritten directly in terms of the mean escape time, i.e. if only the motion between the two halfs of the potentials is considered this can described by this mean escape time completely. The different transition probabilities between the potential wells which are caused by different noise intensities are reflected in the shape of the measures of complexity specifically: Shannon entropy is increasing whereas the Effective Measure Complexity (EMC) is decreasing and the fluctuation complexity shows similar to the Lyapunov-exponents a onehumped behavior. Only the $\epsilon$-complexity is constant for all noise intensities, since it evaluates the topological equivalence of all considered symbol sequences. Hence, the findings for both concepts are in accordance with the well-known ones if that traditional approach is sufficient for 
the description.

2. For the more complicated model with the fluctuating barrier the Lyapunov exponent demonstrates the crossover behavior being a function of the correlation time of barrier fluctuations. Such a simple dependency of the measures of complexity from the mean escape time as in the case of Kramers oscillator does not exist here. Due to long-range correlations in the motion between the halfs of the potentials, it is not sufficient to consider the mean escape time for a complete description. The measures of complexity enable a more detailed view of the structure of those correlations: However, at the correlation time of barrier fluctuations, where the phenomenon of resonant activation occurs, all measures of complexity show an extremum: The Shannon entropy has there its maximum value and the EMC takes its minimum, both is due to the most disordered motion between the two potential halfs. Moreover, SBC has a turning point at this correlation time and two further extrema which mark changes in the relation of the growth rates of Markov order and entropy. The $\epsilon$-complexity indicates the decrease of the Markov order and shows therefore a monotonous behavior.

3. The measures we used can also be applied to study the phenomena of stochastic resonance (SR). In this case the double-well potential is modulated by an external periodic bias. Therefore the output signal contains a periodic component. The Lyapunov exponent being a measure of linear divergence of nearby trajectories is not appropriate measure for SR. So, the only change in the Lyapunov exponent for a bistable system with periodic bias in comparison with the original system we found is that the Lyapunov exponent decreases with the increase of the amplitude of the bias and the value of noise intensity at which the Lyapunov exponent takes its maximum becomes shifted.

By using a full-scale experiment with the Schmitt trigger circuit in [5], it was shown that the source Shannon entropy of the output signal processes a minimum being evaluated versus the noise intensity. This minimum refers to an optimal value of noise level at which SR occurs. We present here the results of calculations of measures of complexity, e.g. the source entropy (calculated with the algorithm of algorithmic complexity) and EMC for numerical simulations of the Schmitt trigger system

$$
y=\operatorname{signum}[\Delta V \cdot y-A \sin (\Omega t)-\xi(t)],
$$

where $\Delta V$ refers to the threshold level of the trigger, $\xi(t)$ is colored Gaussian noise with a cutoff frequency $f_{c}$ and an intensity $D$. We analyze the output of the Schmitt trigger $y(t)$ which is a dichotomic stochastic process. The dependence of the mean switching frequency between states of the trigger (MSF) versus $D$ is shown in Fig. 12 and shows the effect of the MSF locking [28]: in a certain region of noise intensity the MSF is almost constant and equals to the frequency of external bias.

The measures of complexity are shown in Fig. 13. For a very weak noise intensity the switching events between metastable states are very rare so that the system exhibits an intermittent-like behavior. As a result the source entropy is very small. With the increase of noise intensity the MSF increases but still slower than the driving frequency of the bias. The randomness of the output signal increases as well. At the border of the synchronization region, where the MSF becomes matching the frequency of external bias, the fluctuations of the escape times are maximal and as a result the structure of the output signal is most complex: the source entropy takes its maximum while EMC takes its minimal value. After the switching events are synchronized by the periodic force, the source entropy decreases and takes its minimum at the value of noise intensity which corresponds to the maximum of the signal-to-noise ratio (cf. [5, 29]), i.e. to SR. EMC (Fig.13,b) also reflect this behavior: EMC processes a minimum at the border of the synchronization region and a maximum at the optimal noise intensity where SR occurs.

Summarizing, we have shown that the dynamics of stochastic bistable systems can be described completely by Lyapunov exponents and mean escape time only if the system is rather simply structured. For more complex and therefore more interesting systems we emphasize to analyze distributions of local Lyapunov exponents and to apply techniques of symbolic dynamics as appropriate tools for the study of such systems. Measures of complexity allow to describe 


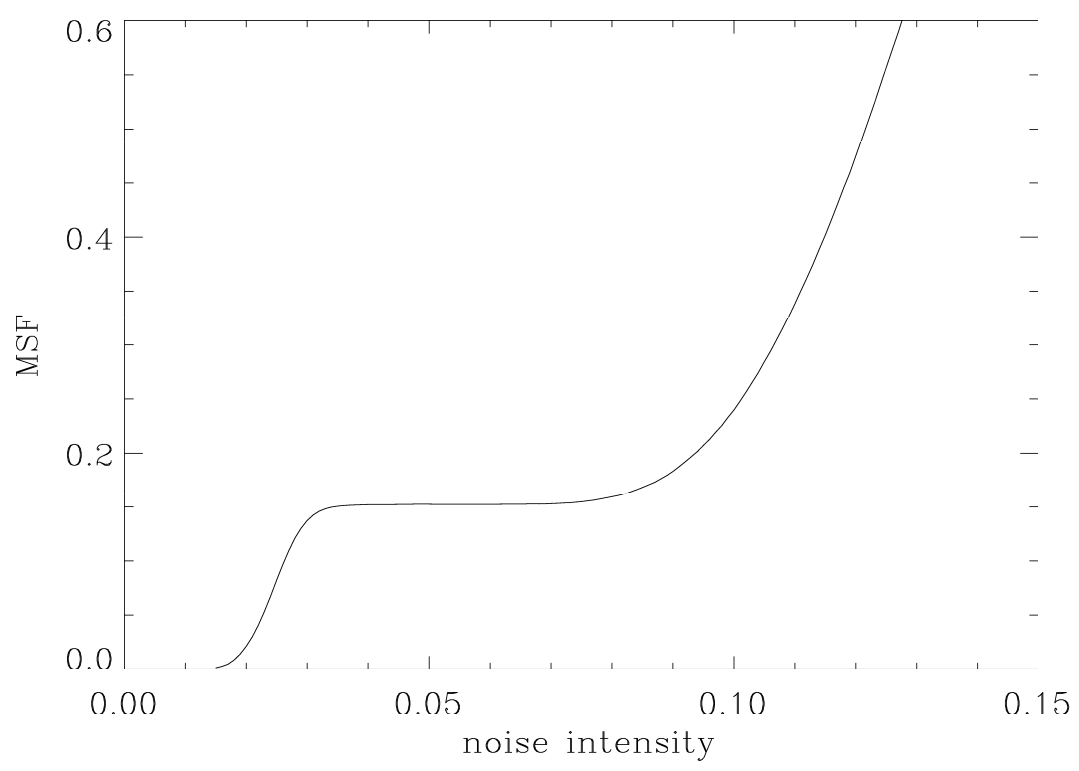

Figure 12: The mean switching frequency vs noise intensity for the Schmitt trigger system (34). The parameters of the trigger are $\Delta V=0.2, f_{c}=100, A=0.15, \Omega=0.5$.

dynamical aspects of the motion between the potential wells and represent, therefore, in comparison with the mean escape time, a more general approach for describing the coarse-grained trajectories. The concept of measures of complexity becomes especially important for experimental investigations, where we have only one observable which often prevents an application of the concept of Lyapunov exponents.

\section{Acknowledgment}

We are indebted for valuable discussions to V.S. Anishchenko, L. Schimansky-Geier, W. Ebeling, A.R. Bulsara, P. Jung, R. Mannella and S. Soskin. A.N. acknowledges warm hospitality during his stay at Potsdam University and support from the Max-Planck-Society and from the Russian State Committee of Science and High School (grant 95-0-8.3-66).

\section{References}

[1] H.A. Kramers, Physica 7, 284 (1940)

[2] P. Hänggi, P. Talkner and M. Berkovec, Rev.Mod.Phys 62, 251 (1990);

V.I. Mel'nikov, Phys.Rep. 209, 1 (1991);

G.R. Fleming and P.Hänggi (eds.), Activated Barrier Crossing. Applications in Physics, Chemistry and Biology (World Scientific, Singapore, 1993).

[3] F. Moss in: Some Problems in Statistical Physics, edited by G.H.Weiss (SIAM, Philadelphia, 1992);

F. Moss, A. Bulsara, and M. Shlesinger (eds.) J. Stat.Phys. 70 (1993) 1-514;

F. Moss, D. Pierson and D. O'Gorman, Int. J. Bifurcation and Chaos 4, 1383 (1994); 

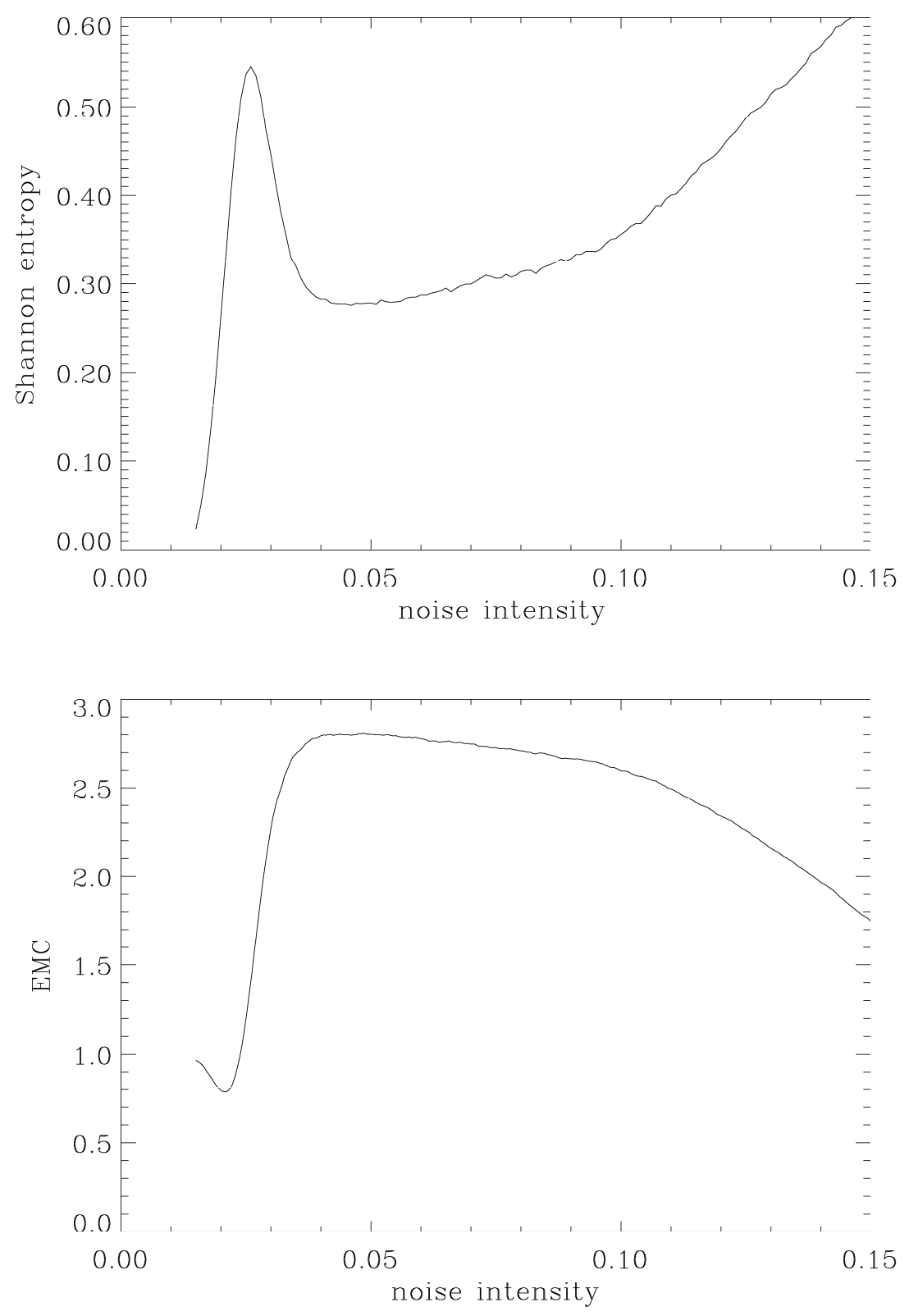

Figure 13: Shannon entropy (a), and EMC (b) versus noise intensity. The parameters are the same as in Fig.12. 
K. Wiesenfeld and F. Moss, Nature 373, 33 (1995);

R. Manella and P.V.E. McClintock (eds.) Proc. 2nd int. Workshop on Stochastic Resonance, Nuovo Cimento D (1995).

[4] Levin and Miller Nature 380165 (1996);

T. Zador and A. Bulsara, Phys.Rev.E (submitted).

[5] A. Neiman, B. Shulgin, V. Anishchenko, W. Ebeling, L. Schimansky-Geier and J. Freund, Phys.Rev.Lett $\mathbf{7 6} 4299$ (1996).

[6] C.R. Doering and J.C. Gadoua, Phys.Rev.Lett. 69, 2318 (1992);

U. Zuercher and C.R. Doering, Phys.Rev.E 47, 3862 (1993);

C. Van den Broeck, Phys.Rev.E, 47, 3862 (1993);

M. Bier and R.D. Astumian, Phys.Rev.Lett., 71, 1649 (1993);

P. Hänggi, Phys.Lett.A, 78, 304 (1980), Chem.Phys., 180,157 (1994);

P. Reimann, Phys.Rev.E, 49, 4938 (1994);

J.J. Brey and J. Casado-Pascual, Phys. Rev. E., 50, 116 (1994)

[7] L.Pontryagin, A.Andronov, and A.Vitt, in:Noise in Nonlinear Dynamical Systems edited by F.Moss and P.V.E.McClintock, Vol.1 (Cambridge University Press, Cambridge, 1989), p.329 (English translation of the original Russian version published in 1933).

[8] C.W. Gardiner, Handbook of Stochastic Methods (Springer-Verlag, Berlin Heidelberg, 1985).

[9] C. Meunier and A.D. Verga, J.Stat.Phys. 50, 345 (1988);

R. Graham in Noise in Nonlinear Dynamical Systems, edited by F. Moss and P.V.E. McClintock, 1225 (Cambridge University Press, Cambridge, 1989)

[10] S. Leibler, Nature 370, 412 (1994)

[11] P. Jung and P. Hänggi, Phys.Rev.A 35, 4464 (1987);

E. Peacock-Lopez, F.J. de la Rubia, B.J. West and K. Lindenberg, Phys. Lett. A 136, 96 (1989)

[12] P. Pechukas and P. Hänggi, Phys.Rev.Lett. 73, 2772 (1994);

A.J.R. Madureira, P. Hänggi, V. Buonomano and W.A. Rodrigues, Phys. Rev. E 51, 3849 (1995)

[13] F. Marchesoni, L. Gammaitoni, E. Menichella-Saetta, and S. Santucci, Phys. Lett. A, 201, 275 (1995)

[14] L. Arnold and V. Wihstutz (eds.)Lyapunov exponents: Lecture notes in mathematics ; 1186, 1486 (Springer-Verlag, Berlin, 1984, 1991)

[15] W. Ebeling, H. Herzel, W. Richert, and L. Schimansky-Geier, Z. Angew. Math. Mech. 66, 141 (1986)

L. Schimansky-Geier and H. Herzel, J.Stat.Phys 70, 141 (1993)

[16] P. Jung, Phys. Rep. 234, 175 (1993)

[17] M. Abramowitz and I. Stegun (eds.), Handbook of Mathematical Functions (National Bureau of Standards, Applied Mathematics Series 55) (1964).

[18] M.C. Mackey, A. Longtin, and A. Lasota, J.Stat.Phys 60, 735 (1990).

[19] R. Wackerbauer, A. Witt, H. Atmanspacher, J. Kurths and H. Scheingraber, "Quantification of structural and dynamical complexity", Chaos, Solitons and Fractals 4, 133-173 (1994). 
[20] A. Witt, J. Kurths, F. Krause, and K. Fischer, "On the reversals of the earth's magnetic field", Geophysical and Astrophysical Fluid Dynamics 77, 79-91, (1995).

[21] J. Kurths, A. Voss, A. Witt, P. Saparin, H. J. Kleiner, and N. Wessel, "Quantitative analysis of heart rate variability", Chaos 5, 88-94, (1995).

[22] Hao, B.-L., "Symbolic dynamics and characterization of complexity," Physica D 51, 611176 (1991).

[23] C.E. Shannon, W. Weaver, The mathematical theory of communication, Univ. of Illinois Press, Urbana (1949)

[24] P. Grassberger, Toward a quantitative theory of self-generated complexity, Int. J. Theor. Phys. 25, 907-938 (1986).

How to measure self-generated complexity, Physica 140 A, 319-325 (1986)

[25] H. Haken, Information and self-organisation, Springer, Berlin (1988)

[26] Hopcroft, J. E. and Ullman, J. D., Introduction to Automata Theory, Addison-Wesley (1979)

[27] J.P. Crutchfield, K. Young, Inferring statistical complexity, Phys. Rev. Lett. 63, 105-108 (1989),

Computation at the onset of chaos, in Complexity, entropy, and the physics of information, Ed.: W. Zurek, Addison-Wesley, Reading, Ma., 223-269 (1989),

J.P. Crutchfield, Inferring the dynamic, quantifying physical complexity, in Measures of complexity and chaos, Eds.: N. Abraham et al., Plenum Press, New York, 327-338 (1989)

[28] B. Shulgin, A. Neiman, and V. Anishchenko, Phys.Rev.Lett. 75, 4157 (1995).

[29] L. Schimansky-Geier, J. Freund, A. Neiman and B. Shulgin, "Noise induced order: stochastic resonance", submitted to the Int. Journal of Bifurcation and Chaos (1996). 